\title{
Why are there descriptive norms? Because we looked for them*
}

\author{
Ryan Muldoon $^{\dagger} \quad$ Chiara Lisciandra $^{\ddagger} \quad$ Stephan HartmanN $^{\S}$
}

July 31,2014

\begin{abstract}
In this work, we present a mathematical model for the emergence of descriptive norms, where the individual decision problem is formalized with the standard Bayesian belief revision machinery. Previous work on the emergence of descriptive norms has relied on heuristic modeling. In this paper we show that with a Bayesian model we can provide a more general picture of the emergence of norms, which helps to motivate the assumptions made in heuristic models. In our model, the priors formalize the belief that a certain behavior is a regularity. The evidence is provided by other group members' behavior and the likelihood by their reliability. We implement the model in a series of computer simulations and examine the group-level outcomes. We claim that domain-general belief revision helps explain why we look for regularities in social life in the first place. We argue that it is the disposition to look for regularities and react to them that generates descriptive norms. In our search for rules, we create them.
\end{abstract}

\section{Introduction}

Our social lives are governed by a large set of formal and informal rules. While we may often only think of formal rules - such as laws and regulations - as constraints on our behavior, informal

${ }^{*}$ The authors would like to thank Kevin Zollman, Jan Sprenger, Conor Mayo-Wilson, and the anonymous referees for their helpful suggestions on earlier drafts of this paper.

${ }^{\dagger}$ Philosophy, Politics and Economics Program, University of Pennsylvania, Philadelphia, Pennsylvania, 19104, USA Email: rmuldoon@sas.upenn.edu.

${ }^{\ddagger}$ Finnish Centre of Excellence in the Philosophy of the Social Sciences, University of Helsinki, Department of Political and Economic Studies, PO Box 24, 00014 Helsinki, Finland

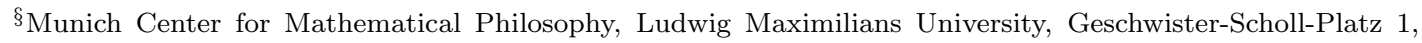
Munich, Germany 
rules have an enormous influence over our behavior, whether we are aware of them or not. One such class of informal rules are descriptive norms. Descriptive norms exist where individuals follow a common pattern of behavior simply because they have a preference for that behavior if they think enough of the rest of the population follows it as well. ${ }^{1}$

Descriptive norms are a curious class of behaviors: unlike social norms, there is no strong normative component. Unlike conventions, they aren't solutions to two-sided coordination problems. ${ }^{2}$ By and large, descriptive norms don't need to exist at all - they aren't solving problems that social groups need solutions to. Even so, our lives are full of descriptive norms. Fashions, fads and all manner of trends fall under the category of descriptive norms. Opinion dynamics, and some ways of expressing political or religious support, can follow the patterns of descriptive norms. These norms continuously, spontaneously emerge from our social interactions. ${ }^{3}$

Precisely because descriptive norms do not represent solutions to problems that social groups face, a distinguishing feature of descriptive norms is they are essentially unconstrained in content. There isn't an underlying fact about the behavioral rules themselves that will guide a population to one descriptive norm versus another, because either is equally good. This suggests that the reasons a particular descriptive norm is 'chosen' is independent of its substance. This is rather different from other previously studied social dynamics, such as the diffusion of a new technology (Young 2009), where features of the choices help guide the pattern of adoption. A new fashion trend isn't typically adopted because of pragmatic features of the new fashion. For instance, 'Brown is the new Black' is a rather different claim than, say, pointing out that word processors make writing and editing documents easier. While some people may prefer brown due to personal tastes prior to any new trend, that is not quite the same as recognizing the innate superiority of brownness over blackness, in the way that we may want to argue that word

\footnotetext{
${ }^{1}$ We follow Bicchieri's definition (Bicchieri 2006). More formally, a descriptive norm is a behavioral rule $\mathrm{R}$ for a population $\mathrm{P}$ in a context $\mathrm{C}$ where individuals in $\mathrm{P}$ have a conditional preference to follow $\mathrm{R}$ if they believe that a large enough proportion of the population $\mathrm{P}$ follows $\mathrm{R}$ in $\mathrm{C}$. This belief is their empirical expectation of rule compliance.

${ }^{2}$ Descriptive norms can be understood either as one-sided coordination problems, or as creating them in a similar manner to the Bicchieri (2006) account of social norms transforming mixed motive games into coordination games. Unlike a convention, which provides a solution to a two-sided coordination game, there is no need to coordinate expectations across parties. One party can simply choose to match what others do.

${ }^{3}$ The distinction between descriptive norms and other informal norms, such as social norms or conventions, however, has fuzzy boundaries. Even in trivial cases, like following a fashion trend, a few individuals might follow them out of fear of punishment or of social rejection, even if no one else would even think of punishing them. Individuals can have a variety of motivations - sometimes one subset of people have normative expectations (the belief that others think you ought to follow the rule), while the rest of the population only has empirical expectations. For our purposes, we focus only on canonical cases of descriptive norms where people lack secondorder normative beliefs.
} 
processors are just superior to typewriters. Quirks of personal taste may leave some in favor of typewriters for a time, but word processors end up taking over because of their efficiency and additional capabilities. In the case of color choice between brown and black, there is no such outside objective measure that can push people to one color or the other. In the same way, a new fashion such as 'Brown is the new Black' is not an instance of a social norm, unlike dress codes. Dress codes such as 'Wear formal attire at ceremonies' involve reciprocal expectations and deviation from them can determine sanctions. By contrast, 'Brown is the new Black' is mainly based on one-way expectations; within the same group there are people who endorse it and others who do not, without the latter incurring the same sanctions they would have had if they had violated social norms. ${ }^{4}$ It is possible that, in the long run, descriptive norms become social norms, and deviations might result in a negative response, but this typically occurs when descriptive norms acquire normative force. In the case of descriptive norms more generally, we can understand the process of change as being governed purely by the process itself. The chosen norms need not offer any advantage over their alternatives. ${ }^{5}$

Other work has examined the emergence of descriptive norms through various heuristic models (Muldoon et al. 2013). But this leaves a challenge: why should we expect those heuristics? Is there some deeper justification that we can find for the social dynamics that we investigated? In particular, can we understand the emergence of descriptive norms as a byproduct of a larger mechanism of hypothesis generation and belief revision and its application to the social domain? Are descriptive norms, then, the spandrels of our larger social-epistemic enterprise?

We propose that the emergence of descriptive norms, and their apparent arbitrariness and instability, can in fact be understood in light of this larger epistemic apparatus combined with an imitation response. In particular, our disposition to find rules stems not from their immediate utility, but rather from the immense value that the general epistemic apparatus has in our lives. That is, while the general disposition to discover regularities and act on the basis of our knowledge of them can be utility enhancing, this does not mean that each instance of rule-following must itself be justified on such grounds. Any given descriptive norm may well be arbitrary, even if the general process that creates them is not.

\footnotetext{
${ }^{4}$ Even if descriptive norms do not involve any normative component, they still differ from pure behavioral regularities, which exclusively depend on individual preferences. A classic example of a behavioral regularity is that people use umbrellas when it rains. They would do this regardless of what other people are doing, namely without being influenced by others behavior, as happens, by contrast, in the case of descriptive norms. Umbrellas are a good solution to a common problem. Color choice doesn't have this feature.

${ }^{5}$ Whereas compliance with social norms can foster group identity and reinforce mutual cooperation, descriptive norms do not necessarily fulfill the same aim, as is shown by the fact that they continually change within the same group: they rapidly emerge, disappear, and come back, while social norms tend to be more stable and long-lasting.
} 
In what follows we will consider an idealized society that could evolve any kind of descriptive norm and we ask: how do some behaviors progressively become entrenched practices and acquire the status of norms? Unlike in previous work, we assume here that agents are Bayesian updaters - they have a domain-general reasoning system that helps them to update their hypotheses about how the (social) world around them operates in light of new evidence.

The specific interest in descriptive norms is twofold: first, these are norms which involve only one level of expectations - namely, what an individual believes the majority of people will do in similar situations. This allows for a formal model of their emergence to remain easily treatable; ${ }^{6}$ Secondly, the philosophical question about this type of norms is why - at least under some conditions - they come to exist and potentially persist, even if there is no objective reason to do so. We approach answering this macro-level question by considering the micro foundations of the phenomenon. How does an agent decide what action to take in an environment that has a variety of options, and social evidence about what others do? When dealing with descriptive norms, the choice of any given particular candidate norm is probably neither right nor wrong per se. There aren't social benefits to adopting one versus another. In cases like these, the evidence provided by other people's behavior becomes the only element upon which to rely. Most interestingly, this evidence does not necessarily bear on the content of the norm, but just the adherence to it. It is purely social evidence. There is no external or objective source of information about the behavioral rule, beyond the actions of others, and this is solely evidence of how others act in accordance with it. ${ }^{7}$ Within an epistemic framework we can analyze the way in which this evidence is processed by agents, given a domain-general updating mechanism that takes other people's behavior into account. Once we associate the outcome of this inferential machinery with an imitation response we can observe the consequences of this process. More specifically, to describe this phenomenon we imagine that an individual finds himself in a situation similar to that of a scientist who is looking for the evidence in support of his hypothesis. As the rational way to proceed in order to estimate the probability of a certain hypothesis about the world is Bayesian

\footnotetext{
${ }^{6}$ Notice once more that, in contrast to other kinds of norms, such as social and moral norms, descriptive norms are behavioral rules which individuals follow on condition that their empirical expectations have been met. By definition, these norms are not based on normative expectations, namely on the belief that others expect us to comply and may sanction deviations. Take as an example the spread of various internet memes, like LOLCats. These memes spread very quickly, and can often fade away just as easily. While many people participate in the meme, there is no particular social sanction for failing to do so. Individual compliance mainly depends on a combination of personal preference and the wish to conform, but it doesn't involve normative expectations about which is the appropriate behavior to follow.

${ }^{7}$ Recall the 'Brown is the New Black' claim versus 'Word Processors are superior to Typewriters' - we can have efficiency measures to compare the machines, but we would be at a loss for an equivalent measure for the colors.
} 
updating, similarly, we express the individual decision problem as a conditional probability.

Here the individual's degree of belief in an action being a regularity is a function of the evidence of other people's behavior and their reliability. The main difference between the adoption of the same inferential system in the natural world and in the social world is that, only in the latter, individuals react to the hypotheses they formulate by complying, at least under certain conditions, with the regularities they detect. ${ }^{8}$ Once the imitative behavior is associated with the Bayesian machinery, we do not have to assume that the individuals have any preexisting notion of a norm of society or any preference for following norms as such. Instead, we are leveraging the fact that a domain-general updating system helps us find regularities in general. In other words, it is our knowledge of regularities in the natural world that triggers the mechanism that prompts us to look for regularities in the social world. However, in the former case, the existence of regularities does not depend on our beliefs and related behaviors, whereas in the latter case social regularities are created via a process in which the more we believe in the existence of the norm, the more we provide evidence in its favor. The social case has evidentiary feedback loops, as we will demonstrate.

We will show how a process can begin such that individual decisions generate evidence that is interpreted by other individuals as an indication of a regularity; the resulting feedback loop can eventually generate the emergence of descriptive norms precisely because individuals were looking for and react to the real or perceived regularities they detect. On this account, our general rule-finding behavior, combined with the special nature of social information leads us to imitate our group members, create the conditions for norms. Norms thus follow from tendencies in our non-social lives, rather than requiring special explanation.

We will proceed in steps. First, we will describe the model of an individual's reasoning that we employ. Next, we will describe a computer simulation that implements this for a group of agents that have some structure to what they can discover socially. We then analyze the results of the simulation, and argue that this model provides evidence that we can make sense of the emergence of descriptive norms if we see it as an instance of a larger cognitive apparatus and its application to the social domain. Finally, we argue that this practice of more domain-general idealized reasoning - using very strong assumptions that likely go beyond our cognitive capacities - allows us to see more universal dynamics across a number of social situations.

\footnotetext{
${ }^{8}$ The systematic tendency to imitate behaviors, which are perceived to be the norm, is a psychological mechanism that has been observed very early in human development. In a family of experiments on social imitation, Tomasello and his research group have shown that infants as young as two years are able to detect social regularities, imitate them and complain against defections, both when the behavior is a means for an intended goal and a goal in itself (See Carpenter et al. (2005), Rakoczy et al. (2008))
} 


\section{The Model}

When studying the emergence of social behaviors, we need a formalism that can account not just for what happens at a group level, but how individual decisions lead to a group outcome. In this study we rely on a Bayesian approach, primarily for its ability to carefully monitor what happens at the individual level. ${ }^{9}$ With a Bayesian model, we can express the individual's degrees of belief about a certain hypothesis and elaborate it with the laws of probability calculus. Broadly speaking, Bayesian epistemology deals with the logic of inductive reasoning and expresses formally how we should learn from experience. ${ }^{10}$

The mathematics of Bayes' rule is straightforward. Its main components are the priors, which measure the probability of the hypothesis before the evidence, and the likelihoods, namely the probability of observing the data given that the initial hypothesis is true. By Bayes' rule, these components are used to compute the posterior probability, i.e. the probability of the hypothesis conditional on the empirical evidence.

As a domain-general updating process, Bayes' rule can be applied to a variety of situations. According to the context, the priors and the likelihoods express the role of different pieces of information for our hypothesis. It can deal with the probability of getting a red ball when drawing from urns filled with balls of different colors, or how much a positive test result should change one's beliefs about whether they've contracted a disease, or in legal settings, how conclusive DNA evidence might be in determining the guilt of a defendant. Across these cases, Bayes' rule shows us how much we can learn from new information and update our previous beliefs to arrive at a new probability of some hypothesis. ${ }^{11}$

In the same way in which Bayes' rule is used to reconstruct an ideal process of updating one's beliefs regarding a certain hypothesis about the physical world, in this paper we adopt it to model changes in beliefs regarding hypotheses about our social world. The way we draw social inferences resembles the more general process of learning from experience. However, in the social world, evidence is interactive: we learn from each other's choices. ${ }^{12}$ To model this situation, we suppose that the members of a group assign a probability to whether a given behavior is an instance of regularity on the basis of the priors, namely the probability of the hypothesis before the observed behavior and of the empirical evidence at their disposal. In this estimate, however, not all evidence is treated equally. We assume that not only are some individuals more

\footnotetext{
${ }^{9}$ Nothing in our argument relies on Bayesian updating in particular - we employ Bayesian updating because it is a well-understood, straightforward model of domain-general belief revision.

${ }^{10}$ See Hartmann and Sprenger (2010) for an introduction to Bayesian Epistemology.

${ }^{11}$ See Hacking (2001) for a formal treatment of the aforementioned examples.

${ }^{12}$ See Schelling (1978) for typical interactive, critical-mass models in the social sciences.
} 
reliable then others (in terms of reliably following norms when present), but also that different individuals are more or less sensitive to other people's behavior.

In what follows, we will formulate a Bayesian model of norm discovery. Our model aims to be a simplified representation of a social situation in which there are multiple behavioral patterns, at most one of which emerges over time as a descriptive norm. To do this, we have eliminated as many superfluous features of real social systems as we could to focus on the core dynamics. The model consists of $n$ agents, representing some pre-existing social group. Within this pre-existing social group are two common behaviors: one which we label $\mathcal{N}$ - the behavioral rule we consider as a possible descriptive norm - and its alternative - the status quo. The model is agnostic about the content of these behaviors. The model unfolds over time. We divide time into discrete rounds and all agents complete the decision process exactly once per round. In what follows, we will formally describe that decision procedure and how it drives the model.

Each agent has beliefs about the state of the world with respect to the proposed behavioral rule. We represent this formally as follows. Each agent has a propositional variable $H$, which can have two values. ' $\mathrm{H}$ ', means that the behavior is a descriptive norm. ' $\neg \mathrm{H}$ ' simply means that the behavior not labeled $\mathrm{N}$ is not a norm. Furthermore, agents can have beliefs not just about the status of the behavioral rule as a descriptive norm itself, but about what others are doing. The variables $E_{i}^{(k)}$ (with $i=1, \ldots, n$ ) have two values: $\mathrm{E}_{i}^{(k)}$ : Group member $i$ follows the proposed behavioral rule in round $k$, and $\neg \mathrm{E}_{i}^{(k)}$ : Group member $i$ does not follow the proposed behavioral rule in round $k$.

In round 0 , everybody is equipped with a probability function $P_{i}^{(0)}$ and starts with a prior probability of $\mathrm{H}$, i.e.

$$
P_{i}^{(0)}(\mathrm{H})=q,
$$

with $i=1, \ldots, n$. We call $q$ the initial belief in the existence of the norm. This is simply the agent's prior belief, independent of what anyone else does. For reasons of simplicity, we assume that all group members have the same priors, but this assumption can easily be relaxed. ${ }^{13}$

On the basis of the prior probability and the epistemic sensitivity (more below on this parameter), group member $i$ will follow $\mathcal{N}$ or not.

At the end of round 0 , each agent considers the behavior of some of the other agents. Let $\mathcal{C}_{i}$ be the set of labels of the group members that group member $i$ takes into account (e.g. the labels of those group members in the visual range of $i$ ). We then define the profile relevant for

\footnotetext{
${ }^{13}$ The assignment of the priors is analogous to the way in which in the natural world we assign priors to our hypothesis before starting to collect evidence in favor of that hypothesis. This assumption does not imply that the individuals have a pre-existing notion of what is in a norm. For instance, the priors can take very low values and it will be the evidence provided by other peoples behavior to trigger the process that can eventually lead to the emergence of a new norm.
} 
group member $i$ in round 0 as $F_{i}^{(0)}:=<E_{k}^{(0)}: k \in \mathcal{C}_{i}>$, with $E_{i}^{(0)}=1$ if $\mathrm{E}_{i}^{(0)}$ obtains in round 0 and $E_{i}^{(0)}=0$ otherwise. Later rounds work in a like manner.

In round 1, each group member updates on the profile relevant to herself, i.e.

$$
P_{i}^{(1)}(\mathrm{H})=P_{i}^{(0)}\left(\mathrm{H} \mid F_{i}^{(0)}\right) .
$$

By Bayes Theorem ${ }^{14}$, we obtain

$$
P_{i}^{(1)}(\mathrm{H})=\frac{q}{q+(1-q) \cdot l_{i}^{(0)}}
$$

with the likelihood ratio $l_{i}^{(0)}$

$$
l_{i}^{(0)}=\frac{P_{i}^{(0)}\left(F_{i}^{(0)} \mid \neg \mathrm{H}\right)}{P_{i}^{(0)}\left(F_{i}^{(0)} \mid \mathrm{H}\right)} .
$$

Let us now calculate the likelihood ratio. To do so, we first introduce the following independence assumption:

$$
E_{i}^{(k)} \Perp E_{j}^{(k)} \mid H
$$

for all $i, j \neq i=0, \ldots, n$ and all rounds $k \geq 0$. The assumption is as follows: If an individual knows that the behavioral rule is a descriptive norm (or not), then (s)he will learn nothing new about the question as to whether group member $i$ follows the norm if (s)he learns that group member $j$ follows the norm. What group member $i$ does depends only on the truth value of $H$. Within a subjective Bayesian framework, the justification for the conditional independence holds as long as people simply assume that the individuals act on the basis of the norm and not on the basis of what others are doing. In other words, it is sufficient that individuals assume conditional independence for the assumption to apply. ${ }^{1516}$

\footnotetext{
${ }^{14}$ See Bovens and Hartmann (2003) ch. 3

${ }^{15}$ It might be asked why an individual should assume conditional independence, given that this assumption is false for her. The main rationale for the independence assumption is based on the psychological motivations underlying the individual decision. Consider again the example of fashion, fads and trends: people usually follow a new fashion trend thinking that they are among the first to do so, not that they belong to what is already a large majority of norm-followers. More generally, individuals have a tendency to disregard their own responsiveness to other people's behavior and to believe that they are acting in conformity with the norm, rather than because others have influenced them. In this sense, the assumption of independence holds at the individual level of the decision-making process.

${ }^{16}$ The actual model makes an assumption of independence across time within an individual, i.e. if an agent observes someone complying with the norm in a certain round this does not affect the probability assigned to that agents complying with the norm in subsequent rounds. The justification for this assumption is that people do not carefully track others inter-temporally, also in virtue of the fact that they have the possibility change their decision from a round to the other.
} 
Next, we assume that

$$
\begin{aligned}
P_{i}^{(k)}\left(\mathrm{E}_{\mathrm{j}}^{(\mathrm{k})} \mid \mathrm{H}\right) & =c \\
P_{i}^{(k)}\left(\neg \mathrm{E}_{\mathrm{j}}^{(\mathrm{k})} \mid \neg \mathrm{H}\right) & =c .
\end{aligned}
$$

Here $c$ is a parameter that measures the expected compliance. That is, it measures to what extent group member $i$ believes that group member $j$ will follow the behavioral rule if it is a descriptive norm, or not follow the behavioral rule if it is not a descriptive norm.

With these assumptions and the definition

$$
T_{i}^{(k)}=\sum_{l \in \mathcal{C}_{i}} E_{l}^{(k)}
$$

where $T_{i}^{(k)}$ denotes how many compliers group member $i$ can see in the $k t h$ round, we can now calculate the likelihood ratio:

$$
l_{i}^{(0)}=\left(\frac{1-c}{c}\right)^{2 T_{i}^{(0)}-n_{i}}
$$

where $n_{i}=\left|\mathcal{C}_{i}\right|$, i.e. the number of group members in the cone of group member $i$.

These equations generalize to later rounds. In round $k+1$, all group members update according to:

$$
P_{i}^{(k+1)}(\mathrm{H})=P_{i}^{(k)}\left(\mathrm{H} \mid F_{i}^{(k)}\right)
$$

By Bayes theorem:

$$
P_{i}^{(k+1)}(\mathrm{H})=\frac{P_{i}^{(k)}(\mathrm{H})}{P_{i}^{(k)}(\mathrm{H})+P_{i}^{(k)}(\neg \mathrm{H}) \cdot l_{i}^{(k)}}
$$

with the likelihood ratio

$$
l_{i}^{(k)}=\left(\frac{1-c}{c}\right)^{2 T_{i}^{(k)}-n_{i}}
$$

In each round $k$, we assume that group member $i$ decides to follow the behavioral rule if $P_{i}^{(k)}(\mathrm{H})>$ $1-s$. Here $s$ is the agent's epistemic sensitivity. However, since the initial behavior is the status quo, the decision to stick to it remains the default option. We assume that the group members continue to update their beliefs even if they are already following the behavioral rule. It is therefore possible that someone who followed the behavioral rule in round $k$ will stop following the behavioral rule in round $k+1$. This is simply to better match the real world - fads can fade away over time. Once a behavioral rule has become a descriptive norm and has full compliance by a given social group, it may be difficult to move away from it, but it does happen with some regularity. ${ }^{17}$ To better capture the possibility of these dynamics, we do not artificially limit the

\footnotetext{
${ }^{17}$ Grunge clothing was popular for several years before it largely disappeared. Bangs are sometimes widely adopted, and then disappear for a while. Text messaging has largely supplanted once-dominant phone calls for quick messages amongst friends.
} 
updating procedure to simply favor norm adoption. In this paper we focus on norm adoption, but if we wanted to better investigate the full lifecycle of norms, including their dissolution, we would consider the possibility of exogenous shocks - like an expansion of the strategy set along with exemplars - or by introducing more noise into agent response rules.

The reader should notice that in this model, each agent applies the standard Bayesian belief revision machinery to the particular case of norm adoption. We suppose that this belief revision machinery is around for other aspects of one's life - it is present in our social reasoning because it is used generally when we reason about the world. We enhance this general model by supposing that there are a few specifically social characteristics of our reasoning that must also be taken into account. These will be discussed in more detail in the next section. However, we note that the small addition of these parameters is all that is necessary to transform the basic reasoning process of Bayesian updating into a social rule discovery mechanism.

\section{Simulating the model}

In order to best explore the model's dynamics, particularly with larger social groups, it was necessary to implement the model in an agent-based simulation. Agent-based simulation allowed us to use a relatively large population (1089 agents), and investigate somewhat more realistic representations of peer influence. For the purposes of this study, we imagine agents to be sitting in a $33 \times 33$ grid, with everyone facing forward. ${ }^{18}$ Each agent sees the agents in its front visual cone (See Figure 1). The intuition behind this representation is that each agent's information and influence is position-dependent. The farther back one is, the more information they have, since they can see more of the other agents. However, the closer one is to the front, the more influence one has, as they are more likely to be seen by others. This structure allows us to represent real-world hierarchical relations in social groups in a general way. This is motivated by work using models of standing ovations as representations of social influence (Miller and Page 2004). ${ }^{19}$ For the purpose of analysis, all simulations were repeated 100 times.

Each agent starts out by following either the proposed behavioral rule or its alternative. This

\footnotetext{
${ }^{18}$ We implemented this simulation in Netlogo 4.0.4. The grid size is the simulation software's default setting. We explored grids of different sizes, and did not see qualitative differences. We report on this population size as a compromise between the desire for a large social group, and the super-linear increase in computational costs (in terms of time) of the simulation as more agents are added.

${ }^{19}$ The assumption of local information and some social hierarchy in our account of how descriptive norms emerge is based on the consideration that full information models are extremely unrealistic very rarely in our social lives do we have complete social information about an entire extended social group.
} 

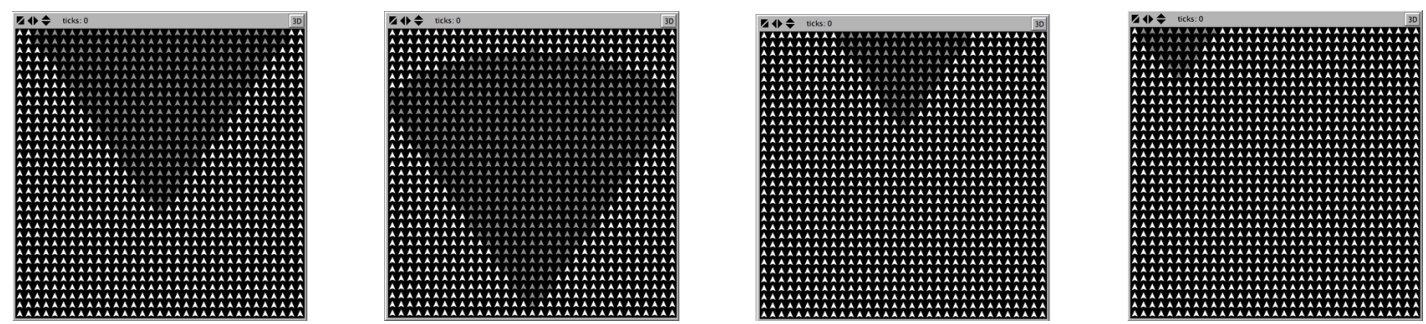

Figure 1: Series of grids representing different agents' visual fields. The grey cones in each grid show the visual field of the agent at the vertex of the cone.

choice, since it relates to a particular action, is fully visible to others. ${ }^{20}$ Because of this, agents can reliably update their beliefs. We investigate what happens as agents update their beliefs over time. Under what conditions should we expect norm emergence? Our investigation revolves around the three free parameters of the model. These parameters augment the standard Bayesian approach by injecting social aspects of our reasoning. These social aspects are the initial belief of an agent about the proposed behavioral rule, an agent's assessment of other agents' expected compliance to the behavioral rule, and each agent's epistemic sensitivity.

More specifically, the initial belief measures the individual opinion about the behavioral rule regardless of other people's behavior. This parameter (combined with the epistemic sensitivity) affects the initial conditions of the model. We use these parameters to determine the initial distribution of agent behaviors. In subsequent rounds, agents update on the evidence provided by other individuals according to the Bayesian procedure described above. If we reflect on the meaning of thes parameter, we ought to expect that by and large the average individual would be fairly neutral between choices of action, since there is no particular utility benefit to either action. In the case of descriptive norms, people's priors should not be that strong in either direction. As such, we should expect that initial belief should be somewhere in the middle of the range from 'absolutely in favor of the behavioral rule' and 'absolutely against the behavioral rule'. In such cases of relative indifference, the other two parameters of the model matter more to an individual's choice: expected compliance and epistemic sensitivity. However, there are scenarios in which we might expect strong individual preferences for given behavioral rules. Due for example to their past experience, individuals might behave according to consolidated practices, that they bring along once in a new group. If the proposed behavioral rule happens to

\footnotetext{
${ }^{20}$ Think of clothing fashions, for example. Descriptive norms, especially ones that have any longevity, have to be associated with some public display or action, otherwise empirical expectations cannot be coordinated. Since there is no normative aspect, there is no reason to a have a descriptive norm about private behavior. Outside of actions influenced by our normative expectations, private behaviors do not have social motivations.
} 
link to an individual's larger set of views or preferences, we might find more extremal values for the initial belief. For instance, some people like wearing plaid shirts, regardless of whether there is a larger grunge fashion trend. Some might think that text messaging is a distasteful form of communication, even if many others use it.

Surely, the most interesting cases are those where individuals start with medium-low initial belief towards a certain norm and end up following it, after having taken into account other people's behavior. Such a decision reflects the fact that agents overcome their initial belief under the influence of some other agents in their network. Thus, our model enables us to distinguish between descriptive norms and behavioral regularities, the latter being those cases where individuals act as they do, not in virtue of other people's influence, but because of their initial preferences. With an example, we might have an entire population wearing white socks, not because it is fashionable, but simply because individuals each like white socks. This behavior, however, is not an instance of descriptive norms, which instead emerge when individuals initially do not have a strong preference towards certain behaviors, e.g. wearing white socks, but start following them after having seen other people doing so. ${ }^{21}$

The expected compliance parameter measures the reliability that each agent assigns to the members of the group. It indicates if the source of evidence matters for the decision of whether to follow the norm. In other words, if an individual is assigned high expected-compliance and the behavioral rule is starting to look like a descriptive norm, then that individual will follow it. In real-world scenarios, this corresponds to reliable or influential people who, for whatever reason, are considered to be competent on that matter. Hence, agents who have been assigned high expected compliance will be judged as reliable indicators of the presence of a norm if they are following it and of the behavioral rule's failure of becoming a norm if they are not following it. This helps capture the idea that we have potentially different assessments of the same evidence (following the behavioral rule) when it comes from different sources (more or less reliable trendsetters).

The epistemic sensitivity parameter measures an agent's individual degree of responsiveness to perceived empirical regularities. In other words, the epistemic sensitivity parameter is the means by which agents convert their epistemic state into a motivation for action. For instance, in a non-social case, epistemic sensitivity determines whether an agent would act on her belief that a particular river floods with some regularity. This may lead her to build her house farther away from the river's banks. In the social case, it reflects the fact that some agents are more

\footnotetext{
${ }^{21}$ We do not specifically look at cases where group behavior contrasts with individuals' preferences, insofar as our interest here mainly consists in analyzing the updating mechanism that leads people to assess other people's behavior as a sign of the existence of a norm and behave accordingly. For a more detailed treatment of this case, consult Muldoon et al. 2013.
} 
responsive to social cues than others. The epistemic sensitivity parameter measures the impact of the evidence on the individual decision as to whether to follow a norm. Some people match their behavior to perceived behavioral rules. Others see the social regularities, but they are more cautious before changing their behavior. Most people fall somewhere in the middle - we may care what others do, but it isn't our only concern.

The epistemic sensitivity parameter as used in this model is paired with an assumption of an imitation dynamic. This dynamic reflects a psychological mechanism characterized as the tendency to imitate other people's behavior, when it is perceived as an instance of a social regularity. This tendency has been observed in children as young as two years old (Rakoczy et al. 2008) and is considered to be indicative of a social learning process, in which the endorsement of arbitrary norms takes precedence over considerations of content or utility. While a more critical stance on the role of norms and social conventions emerges later on in development (Turiel 1983), studies on imitation in the field of social cognition seem to suggest that a domain-general rule-following behavior is part of our psychological endowment (Carpenter et al. 2005) and is dedicated to the acquisition and development of normative behavior. Descriptive norms might be a case in point where this mechanism is triggered, when there are no other considerations that advise against their adoption.

Note that imitation alone would not be enough to explain the overall phenomenon of descriptive norms. Imitation by itself doesn't differentiate between social and individual behavior. Only certain behaviors are considered to be instances of social rules and to account for this feature we need an inferential system that formulates hypothesis about the social world, and assign probabilities to events and their likelihood; A model of pure imitation differs from the Bayesian one, insofar as it lacks a parameter that takes into account peoples reliability and the priors, and updates on them. Pure imitation does not allow for the case where the decisions of the individuals are anti-correlated when their reliability is low; nor for a situation in which a few reliable individuals have a stronger impact on other peoples decisions. ${ }^{22}$ More generally, a Bayesian machinery offers a more nuanced representation of the imitation dynamics, which reflects the way in which these processes usually occur in society. It is rarely the case that we only base our behaviour on past observations, or that we repeat the last thing we saw someone else doing. More often, different individuals are differently influenced by other peoples behavior, according to their reliability and position in society. The Bayesian likelihood is particularly appropriate to capture such aspects of social conditioning. Imitation by itself is too simplistic, but it can be

\footnotetext{
${ }^{22}$ All evidence being equal, in a Bayesian model the likelihood affects the judgment about whether the behavior in question is an instance of a norm; by contrast, in a pure imitation model, the emergence of the norm depends exclusively on the number of followers (if we consider the mode), regardless of their influence on each other.
} 
quite powerful when paired with a more general inference mechanism.

However, one might ask why imitation is privileged over other considerations: why not a best response dynamic, for instance? Our reason for selecting imitation over other choices is that imitation does not require knowledge of the structure of the game. The full strategy set is not transparent, nor are the payoffs of the strategies. The agents do not even know if the payoffs are differential. ${ }^{23}$ Consider again the example of fashion or Internet memes. No agent has access to the full strategy set. What are all possible fashion choices? What are even the active fashion choices currently on display in the United States? It is difficult to even retrospectively determine what the total set of fashion choices made was in a set time and place. Internet memes and other fads pose a similar challenge. The strategy space is simply enormous. Given this basic fact, we are severely constrained by what our options are to convert our beliefs into actions. I can imitate without knowing about the full strategy set, but I cannot best reply to what others do if I am unaware of the strategy set. In other words, it is unknown to the individuals what is the game they are playing, if they are playing a game at all. Since we argue that descriptive norms can come about when we are trying to learn about uncertain situations, it would be questionbegging to explain them as a mistaken interpretation of potentially advantageous norms. We would be assuming what we want to explain if we claimed that we create descriptive norms on the basis of the advantages associated with them, in the same way in which, for example, it would be question-begging to explain how people started to use a certain grammar once they had a language to determine it. The benefits - if there are any - become clear once the norm has spread in the group, after people have started to endorse them even before any consideration of utility.

To close our defense of the choice of an imitation rule, recall the scenario that we consider. An agent has come to believe that she has discovered a social rule by observing what others do. She might simply not care, because she's just not that interested in what others do. But if she does care, then following the rule is what seems to fit in with our normal psychology. We agree that some small portion of the population has a contrarian tendency, but not a big enough number of people to matter. If such a psychology dominated, then our model would fail to ever get off of the ground - no regularity would ever come to pass, or at the very least, it would fail to persist. Since we empirically observe the existence of descriptive norms, it must be the case that the relevant dimension is how socially sensitive people are, on a scale from indifferent to eager to imitate.

\footnotetext{
${ }^{23}$ It is not necessarily the case that, when there are no differential payoffs, the individual believes that there are in order to choose between the possible alternatives. Think for example of conventions, where a decision can be taken despite equal benefits between alternatives.
} 
The main predictions of the model can be summarized as follows:

1. Norm emergence is incompatible with very low priors. If agents strongly disbelief that a behaviour is an instance of a behavioural rule, then they will not follow it. More generally, this means that norm emergence is not guaranteed in the mathematics of the model. Not all behavioral rules become descriptive norms.

2. Other things being equal, the higher the epistemic sensitivity, the more probable that the proposed behavioral rule will in fact become a descriptive norm.

3. The agents' decisions correlate with those agents to whom they assign high-expected compliance. Otherwise, decisions are anti-correlated with one other. In a group of people where all agents have low reliability, the decisions will fluctuate between opposite choices, in this way precluding the possibility of the emergence of the norm.

4. The higher the expected compliance, the more the evidence weighs in favor of or against the hypothesis. In other words, if agents are considered to be highly reliable, their behaviors will have an higher impact on other people than those of less reliable people. This means that it takes fewer more reliable agents for the emergence of a norm than of less reliable agents.

To examine these predictions, we ran a series of simulations to experiment with the effect of the parameters. We present the results in the following subsections. In the next section, we will consider how the model does at providing a general explanatory framework for the phenomena of norm emergence.

\subsection{Simulation results}

The results of the simulations show under which conditions a descriptive norm emerges, under which it doesn't, and how the parameters and their interplay affect the final outcome. In the first group of simulations, we analyzed descriptive norm emergence as a function of the agents' initial belief. As expected, the higher the priors, the higher the probability that the hypothesis under consideration holds true. In our study, this is reflected by the fact that the probability for descriptive norm emergence increases for higher values of the initial beliefs, as shown in Figure 2. We see that full convergence on the candidate behavioral rules obtains for high values of the priors combined with moderate to high values of expected compliance and epistemic sensitivity. With lower priors, the percentage of individuals following the norm decreases proportionally. This is what we expected from the mathematics of the model and expresses the idea that if 
the preference for the behavior is low then the chance that it spreads are low as well. This is unsurprising - more preferable behaviors are more likely to spread in a population. Less preferable behaviors have a harder time. We may pay attention to what others do and change our behavior to be more compliant, but we have our limits.

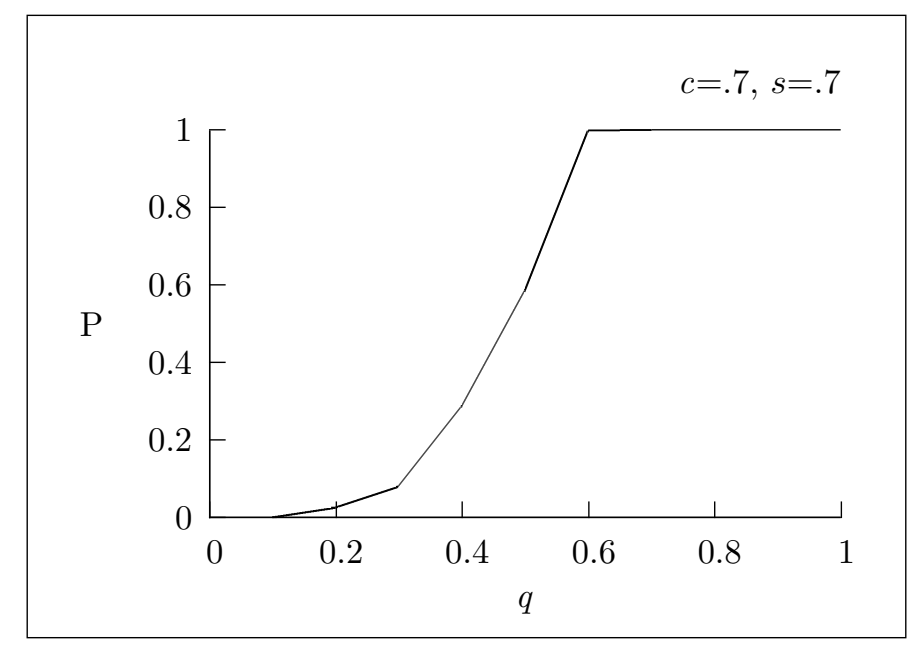

Figure 2: Probability $P$ of a norm to emerge as a function of the initial belief q combined with medium expected compliance $(\mathrm{c}=.7)$ and medium epistemic sensitivity $(\mathrm{s}=.7)$.

In the second group of simulations, we analyzed the conditions for norm emergence as a function of the expected compliance parameter. By Bayes' rule, the likelihood affects the estimate of a certain hypothesis in such a way that the higher its value, the more significant the evidence is for the hypothesis at stake. In our study, this is reflected by the fact that descriptive norms emerge when reliable individuals follow the behavioral rule and they do not emerge when unreliable individuals follow the behavioral rule. To study the role of the expected compliance parameter on the individuals' decisions over time, we monitored the posterior probability of a few spatially randomized agents, from the beginning of the simulation to the equilibrium point. The simulations results showed that agents tend to follow the norm when the expected compliance parameter is high and it is combined with high value of initial belief. By contrast, other simulations showed that agents do not follow the norm when the expected compliance parameter is combined with low priors. In both cases this happens because everyone considers other people's behavior to be highly dependent on the truth or falsity of the hypothesis. Hence, they only follow the behavioral rule if enough other people follow it, and they don't otherwise, suggesting that only in the former population does the behavioral rule become a descriptive norm.

We find that the expected compliance parameter has a large influence over the model. Note that the tipping point is when the parameter is at 0.5 . When the parameter is in the range $(0.5$, 
1], agents count other agents' behavior as significant evidence for or against the hypothesis. As the parameter value trends towards 1 , it is harder for a norm to emerge. This is a counterintuitive result of the model that is worth stressing. As expected compliance ramps up, any time we see someone not following the behavioral rule, that is considered to be significant adverse evidence. If people are reliable indicators, and someone is not following the behavioral rule, then we surmise that the rule hasn't become a descriptive norm. Evidential standards get more stringent when we perceive the data to be less noisy. Adverse behavior is less likely to be a fluke, and is instead interpreted as evidence that there is no norm. We illustrate this phenomenon in Figure 3, where the probability of the norm is given as a function of the priors and the expected compliance parameter.

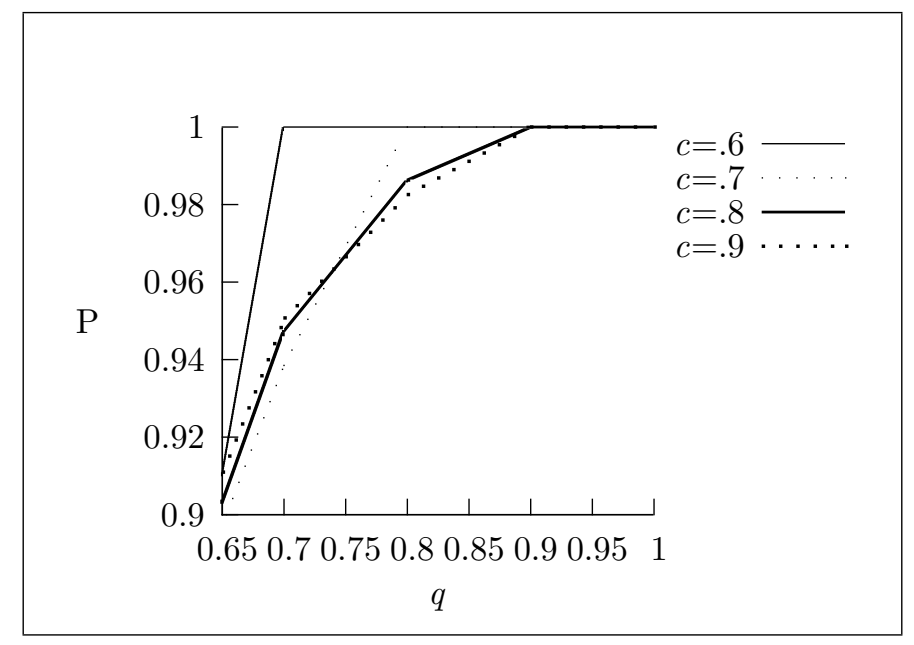

Figure 3: Probability $P$ of a norm to emerge as a function of the initial belief q combined with increasing values of expected compliance $(\mathrm{c}=.6, \ldots, \mathrm{c}=.9$ and medium epistemic sensitivity).

The initial belief is represented on the $x$ axis. The $y$ axis tracks the number of agents following the behavioral rule. We plot different values of the expected compliance parameter on the same set of axes to compare them. We see when the expected compliance parameter is lower, agents need to see fewer agents following the behavioral rule to comply with it themselves. ${ }^{24}$

Finally, we used the simulation to explore the effects of the epistemic sensitivity parameter. In the model there is a gap between discovering whether the behavioral rule is a descriptive norm and the decision to follow it. The same probability estimate can induce an epistemically sensitive

\footnotetext{
${ }^{24}$ In a further extension of the model, we intend to include a number of non-conformists, who react negatively to the emergence of a norm. In this scenario, the expected compliance parameter would be negatively correlated to the existence of the norm: positive evidence by a non-conformist suggests that there is no norm and negative evidence that there is a norm.
} 
agent to follow the norm and a less sensitive one not to follow it. Keeping other things equal, we see that when the value of the epistemic sensitivity is low, there is never norm emergence. Social information is simply irrelevant. When instead the epistemic sensitivity is high, at the end of the simulation all agents follow the norm. In other words, for the same probability assigned to the hypothesis that the behavioral rule is a descriptive norm, the difference in epistemic sensitivity induces agents to consider or ignore this information. ${ }^{25}$

Recall that, in the present model, we assume that all individuals have the same parameter values. However, in the real world, there are many ways in which agents can differ from one another and in their evaluation of social evidence. For example, they might have different epistemic sensitivities, i.e. not respond equally to the same social evidence, or they might have different levels of expected compliance. In future works, we intend to explore heterogeneous groups by introducing different kinds of agents: for example, those who are indifferent to their social niche or non-conformists, who react negatively to the diffusion of descriptive norms. Also, we want to observe the effect of different individuals having different reaction times to the same information, where the probability of responding to social evidence decreases as time goes by. We predict that these variations of the initial model will have the effect of modulating the process of the emergence of norms and slowing convergence down. In other words, these extensions will refine the initial predictions by limiting the number of situations where a descriptive norm emerges, thereby better accommodating the evidence that it is only under certain conditions that we see descriptive norms emerging in a group.

Overall, the results presented in this section combine to show the conditions under which Bayesian agents will come to decide that a behavioral rule is in fact a descriptive norm and comply with that behavior. In summary, the simulations help to illustrate to what extent the individual preference matters for the emergence of the norm, and how reliable and socially sensitive individuals affect the process. ${ }^{26}$

Simply relying on a domain-general belief revision mechanism and an imitation dynamic, we can generate rather complex social behavior. As we should expect from our everyday experience

\footnotetext{
${ }^{25}$ The tipping point after which the value of the epistemic sensitivity determines the emergence of the norm depends on the combination of the values of the other two parameters. It would be interesting to bound the value of the epistemic sensitivity parameter further, in the light of the costs that conformity has in terms of money and work. We predict that also this variation of the model would provide a limiting mechanism to the emergence of descriptive norms.

${ }^{26}$ Several mechanisms might determine the decline of a norm. Two clear candidates are that either a new norm emerges and people switch to it or that an old norm simply fades over time. The former option can serve as a useful description of fashion cycles, while the latter option is particularly clear in the case of fads - eventually they just get old and unexciting. Since we are focusing on norm emergence we leave out considerations of norm decay in order to reduce the complexity of the model.
} 
with descriptive norms, not every behavioral rule becomes a descriptive norm. Actions that most people don't like much rarely if ever become descriptive norms. When we think people are only moderately reliable in detecting whether a behavior is a descriptive norm or not, it's more likely that we pick out patterns in the noise that might not have been there in the first place. In these results, we find qualitative agreement with results from previous heuristic models (Such as Muldoon et al. 2013), but as we will discuss in the following section, we claim that this approach can offer a deeper explanation of the phenomena.

\section{Using the Model as a Unifying Explanation}

When we consider how to model complex phenomena like norm emergence, there are two different (and complementary) approaches that can be pursued. First, we might want to look at the proximate reasons for norm emergence. Given some assumptions about how we behave in social situations, how do norms come about? Here, heuristic models are often going to be particularly useful. Heuristic models (such as Muldoon et al.) present cognitively realistic mechanisms for norm emergence. These heuristic models let us consider the world of boundedly rational agents, and how, despite these limitations, they can systematically create new descriptive norms when the conditions are appropriate. What these heuristic models cannot do, however, is motivate themselves. Nothing internal to the model can tell us why people might track what others do and treat that as evidence for what they themselves should do. Heuristic models - by design can only speak to proximate causes, not ultimate causes. They do not attempt to ask questions broader than proximate explanation.

Descriptive norms, since they are devoid of any normative force, could seem quite strange if we just look at them with heuristic models. We know that descriptive norms exist, and heuristic models help us understand how they form, given that we're the sorts of agents that look for social rules to follow. But why we are the sorts of agents that have descriptive norms at all goes unanswered. It is only when we turn to a more domain-general style of modeling that we can see that descriptive norms are a side-effect: they are the accidental combination of our system for belief updating and the propensity to imitate in the social realm. This second approach to modeling complex phenomena offers a deeper explanatory framework. Here, rather than focusing on the details of an individual's thought process, we can ask ourselves if there is a more general explanation for the pattern of behavior. Namely, can we help explain why agents look for rules of behavior to follow in the first place? In particular, is there a way of explaining this phenomenon by demonstrating its connection to mechanisms we understand in other areas of science? Reconstructing the general epistemic process helps us understand the 
pattern of behavior, even if it does not necessarily capture the precise details of an individual's cognitive processes. What's more, by relying on idealized models of epistemic updating, such as Bayesian updating, we can more easily see how the phenomenon under study can be understood as instances of a broader framework that has shown success elsewhere. Bayesian reasoning is domain-general, and has been used to examine problems across epistemology and the philosophy of science (see Chater and Oaksford (2008), Griffiths et al. (2010), Tentori et al. (2007), Schupbach (2011)). ${ }^{27}$ Though we may lose a bit in our proximate explanations of individual behavior, by not focusing on the details of the decision processes of cognitively limited agents, our more general reconstruction of this behavior allows us to see how descriptive norm emergence relates to a wider field of epistemic practice.

Bayesian models have seen much success in showing how we can learn about nature, as we come across new evidence (see Jones and Love (2011) for a critical review on the literature). The general process of hypothesis formation, testing, and updating on evidence is well-established in philosophy of science. Even children seem to be successful at learning about causal properties and law-like regularities this way (Gopnik and Tenenbaum (2007), Gopnik et al. (2004)). Bayesian belief revision's success in dealing with the natural world provides a reason why we might find individuals naturally extending this apparatus to the social world. Rather than just suppose that people do update beliefs based on social information, we can say something about why they update beliefs based on social information. In the absence of any norms, there would be no social rules to follow, so no reason to motivate the responsiveness that we have to social cues. However, if we suppose that this responsiveness comes from a domain-general updating mechanism, then we only need to rely on its proven success in other domains. The benefit we get from knowing law-like regularities in the physical world provides a reason to suppose that there will be benefit from discovering norms in the social world. Most of the time, the responsiveness that we have to social cues manifests itself in the adoption of the perceived norm. In this sense, we take the imitation response to be the natural learning rule for this context. The Bayesian model offers an alternative explanation to the heuristic model, albeit the results of the two approaches are largely consistent. But the Bayesian model has an advantage. It enables us to explain the emergence of descriptive norms as the result of an updating process which resembles the way in which we discover regularities in the natural world. This, in turn, favors the hypothesis that there might be the same underlying mechanism at the basis of the discovery of regularities in the natural and

\footnotetext{
${ }^{27}$ Again, we would like to note that Bayesian reasoning here is just an exemplar of domain-general reasoning about hypotheses and their evidence. Nothing hinges on Bayesianism in particular. Which means, if it turned out that a different inferential system were a more accurate representation of the individual reasoning process, then we would have to show how to adapt that system to the social domain, in order to see under which conditions it would explain the emergence of descriptive norms.
} 
in the social world.

Overall, it is the success in other domains that explains why we would see a domain-general updating mechanism applied to social cases. Agents are already accustomed to employing such cognitive machinery in a wide variety of cases, and so the social case is just one more instance of using the same basic tools. If anything, it would seem strange to adopt a different epistemic method than the one used so widely in other aspects of one's life. By looking at a wider scope of human activity, we can better see how apparently unrelated tasks can shape our responses in novel situations. The benefit of doing this is that we find that we can get at a more substantive explanation for norm emergence than what is offered in heuristic modeling. In particular, we show that descriptive norms can emerge even if agents are indifferent between a world with no norms and a world full of them. However, the agents do look for regularities. In the same way in which we rely on empirical observation to uncover law-like regularities in the natural world, we extend the same reasoning system to the social world. In the natural world, the discovery of physical laws is a process of continuous refinement and revision: almost never we are neutral observers; we assume certain hypotheses, we behave as if they were true, in a way that brings us to the discovery of new and unexpected regularities. Similarly, in the social world we react to the real or presumed regularities we identify. However, the social situation is unique in that by looking for regularities, regularities are created. If the agents did not try and update their beliefs, and act in accordance with those updated beliefs, norms could never emerge. Unlike private behaviors, norms are public. They are things that we do because others do them. Norms require social expectations. Since descriptive norms have no particular intrinsic value - they don't solve crucial pre-existing coordination problems, they don't improve public morality, and they could easily have been otherwise - they can only come about if enough people believe that they were already there and if they are socially responsive. For beings like us with the psychological tendencies we exhibit in the social world, rule discovery triggers an interest in following the rule. Once this process begins, norms can start to emerge. In this sense, they are created out of nothing, but become real enough once the individuals start to believe they hold true.

We can see this clearly in the dynamics of the models themselves. In the initial conditions of the model, there is no norm. But simply in virtue of looking for a pattern in what others do, we start getting a pattern in virtue of more correlated behavior. Once that correlation gets off the ground, the more the agents observe and update, the more they start acting in accordance with the apparent norm. This updating system creates a positive feedback loop. The feedback loop doesn't always start - there aren't always the right conditions for it - but once it does get going, a norm comes to be purely because people were looking for it, and choose to follow the 
rules they perceive themselves to have found.

In fact, our system of belief revision will, if anything, overreact to social evidence. In the natural world, when we observe a piece of evidence for, say, whether the moon is waxing or waning, our observation does not effect the moon, nor the observations of others. In the social world, however, if Bob and Carol see Alice change behavior on Monday, and because Bob sees Alice change her behavior, he updates and changes his behavior as well, Carol might now also update on Bob's behavior. But that would just be counting the first piece of information twice. We naturally assume independence amongst agents in normal situations. Unless we have a good reason for supposing that other people's behaviors or beliefs are correlated, we conceive of other people as making their own decisions. This premise is often true, but can be a catalyst in social phenomena such as norm emergence - the incorrect assumption of independence can lead to large scale behavior change because the first movers have far more influence than people think they have. $^{28}$

Explaining descriptive norm emergence in terms of ultimate, rather than proximate explanations also allows us to think more systematically why we see the norms we do. If we focus on proximate explanations, we run the risk of having to rationally justify each norm. We may get caught up in trying to find ways to argue that each individual norm is utility-enhancing in the same way that the transition from typewriters to word processors enhanced our utilities, rather than the much more defensible claim that the general epistemic processes that have spawned descriptive norms are utility-enhancing. We have argued that the system of belief revision has proven itself to be a massive asset, and so it would naturally be extended to the social realm. By moving away from proximate explanation, and moving toward ultimate explanation, we can come to understand why descriptive norms emerge. Not because of anything that they do for us, but because they come about from a process that's valuable to us in other areas of our lives.

\section{Conclusions}

As we have seen, once we look at descriptive norms through the lens of a purely epistemological procedure, transformed into a social context, we can see why they can come to be, and persist, even if any particular descriptive norm has no particular value. The process of norm emergence comes along for the ride once we have a general framework for belief revision, and a psychology that primes us for rule following. As such, we ought to expect (and in fact find) an accumulation of descriptive norms. This itself can reinforce further norm emergence. Once we are in an

\footnotetext{
${ }^{28}$ As discussed earlier, it is in part due to this insight that we chose the network structure that we did for our simulations.
} 
environment where we are aware that there are already a lot of norms, then it is rational to be all the more vigilant for finding more. This can make us increasingly sensitive to norm discovery. So, while descriptive norms may have started out as a mistaken application of a domain-general belief revision system, their accumulated presence provide a justification for why that belief revision system ought to be applied to them after all. Once our social environment includes descriptive norms as one if its elements, then we have good reason to search for norms as we survey our social world. Descriptive norms may have come to be through a mistake, but their accumulation created a self-justifying reason for their existence. 


\section{References}

Bicchieri, C. (2006) The Grammar Of Society. New York, NY: Cambridge University Press

Bovens, L. and Hartmann, S. (2003) Bayesian Epistemology. Oxford: Oxford University Press

Carpenter, M., Call, J., and Tomasello, M. (2005) Twelve- and 18-month-olds copy actions in terms of goals. Developmental Science 8: F13-F20

Chater N. and Oaksford (2008) The Probabilistic Mind. Oxford: Oxford University Press

A. Gopnik and J. Tenenbaum (2007) Bayesian networks, Bayesian learning and cognitive development. Developmental Science (special section on Bayesian and Bayes-Net approaches to development) 10(3): 281-287

Gopnik, A., Glymour, C., Sobel, D., Schulz, L., Kushnir, T., \& Danks, D. (2004). A theory of causal learning in children: Causal maps and Bayes nets. Psychological Review 111(1): 3-32

Griffiths, T. L., Chater, N., Kemp, C., Perfors, A., \& Tenenbaum, J. B. (2010) Probabilistic models of cognition: Exploring representations and inductive biases. Trends in Cognitive Sciences 14(8): $357-364$

Hacking, I. (2001) An Introduction to Probability and Inductive Logic. Cambridge UK: Cambridge University Press

Hartmann, S. and Sprenger, J. (2010) Bayesian Epistemology. in: S. Bernecker and D. Pritchard, eds., Routledge Companion to Epistemology London: Routledge: 609-620.

Jones, M. \& Love, B.C. (2011) Bayesian Fundamentalism or Enlightenment? On the Explanatory Status and Theoretical Contributions of Bayesian Models of Cognition. Behavioral and Brain Sciences 34: 169-231

Miller, J. \& Page, S.E. (2004) The Standing Ovation Problem. Complexity 9(5): 8-16.

Muldoon, R. Lisciandra, C. Bicchieri, C. Hartmann, S. Sprenger, J. (forthcoming) On the Emergence of Descriptive Norms. Politics, Philosophy, and Economics

Rakoczy, H., Warneken, F., and Tomasello, M. (2008) The sources of normativity: Young childrens awareness of the normative structure of games. Developmental Psychology 44(3), 875881

Schupbach, J. N. (2011) Comparing Probabilistic Measures of Explanatory Power. Philosophy of Science 78(5): $813-829$ 
Schelling, T. C. (1978) Micromotives and Macrobehavior. New York: W.W. Norton

Tentori, K. Crupi, V. Bonini N., and Osherson D. (2007) Comparison of Confirmation Measures. Cognition 103(1): 107-119

Turiel, E. (1983) The development of social knowledge: Morality and convention. Cambridge: Cambridge University Press.

Young, H. P. (2009) Innovation diffusion in Heterogeneous Populations: Contagion, Social Influence, and Social Learning. American Economic Review 99(5): 1899-1924 